University of Nebraska - Lincoln

DigitalCommons@University of Nebraska - Lincoln

2018

Focused electron-beam-induced deposition for fabrication of highly durable and sensitive metallic AFM-IR probes

Wen Qian

Shuo Sun

Jingfeng Song

Charles Nguyen

Stephen Ducharme

See next page for additional authors

Follow this and additional works at: https://digitalcommons.unl.edu/physicsducharme

Part of the Atomic, Molecular and Optical Physics Commons, and the Condensed Matter Physics Commons

This Article is brought to you for free and open access by the Research Papers in Physics and Astronomy at DigitalCommons@University of Nebraska - Lincoln. It has been accepted for inclusion in Stephen Ducharme Publications by an authorized administrator of DigitalCommons@University of Nebraska - Lincoln. 
Authors

Wen Qian, Shuo Sun, Jingfeng Song, Charles Nguyen, Stephen Ducharme, and Joesph A. Turner 


\title{
Focused electron-beam-induced deposition for fabrication of highly durable and sensitive metallic AFM-IR probes
}

\author{
Wen Qian, ${ }^{1}$ Shuo Sun, ${ }^{2,3}$ Jingfeng Song, ${ }^{2}$ Charles Nguyen, ${ }^{1}$ \\ Stephen Ducharme, ${ }^{2}$ and Joseph A Turner ${ }^{1}$
}

\author{
1 Department of Mechanical \& Materials Engineering, University of Nebraska- \\ Lincoln, Lincoln, Nebraska NE, United States of America \\ 2 Department of Physics \& Astronomy and Nebraska Center for Materials and \\ Nanoscience, University of Nebraska-Lincoln, Lincoln, Nebraska NE, United \\ States of America \\ 3 State Key Laboratory of Infrared Physics, Shanghai Institute of Technical \\ Physics, Chinese Academy of Sciences, 500 Yu Tian Road, Shanghai, 200083, \\ People's Republic of China \\ Correspondence - W. Qian wqian2@unl.edu and J. Turner jaturner@unl.edu \\ ORCID iDs \\ Wen Qian https://orcid.org/0000-0001-5813-7817 \\ Charles Nguyen https://orcid.org/0000-0001-7649-8384
}

\begin{abstract}
We report on the fabrication of metallic, ultra-sharp atomic force microscope tips for localized nanoscale infrared (IR) spectrum measurements by using focused electronbeam-induced deposition of platinum or tungsten. The tip length can be controlled by changing the duration time of the electron beam. Probes of $12.0 \pm 5.0 \mathrm{~nm}$ radiusof-curvature can be routinely produced with high repeatability and near-100\% yield. The near-field-enhancement appears stronger at the extremity of the metallic tip, compared with commercial pristine silicon-nitride probe tip. Finally, the performance of
\end{abstract}

Published in Nanotechnology 29 (2018) 335702 (7pp)

doi:10.1088/1361-6528/aac73c

Copyright (C) 2018 IOP Publishing Ltd. Used by permission.

Submitted 9 February 2018; revised 16 May 2018; accepted 23 May 2018; published 8 June 2018.

Supplementary material follows the References. 
the modified metallic tips is demonstrated by imaging PVDF and PMMA thin films, which shows that spatial resolution is greatly enhanced. In addition, the signal intensity of the localized nanoscale IR spectrum is increased offering greater sensitivity for chemical IR imaging.

Keywords: electron-beam-induced deposition, AFM, localized infrared spectrum, chemical mapping, metallic probe tip

\section{Introduction}

Atomic force microscopy-based infrared spectroscopy (AFMIR) is a rapidly emerging technique that provides chemical analysis and compositional mapping with spatial resolution, which is far beyond conventional optical diffraction limits [1-3]. AFM-IR works by using the tip of an AFM contact probe to detect the local thermal expansion in a sample resulting from absorption of IR radiation. Therefore, it can provide nanoscale spatial resolution, which is effectively limited only by the AFM tip apex size $[4,5]$. Coupling the 'lightning rod effect' $[6,7]$ with resonance enhanced AFM-IR has enabled nanoscale infrared (nanoIR) spectroscopy and chemical imaging on organic materials, polymers and materials in life sciences [8].

Currently, commercial AFM-IR probes are micro-fabricated silicon-nitride ( $\mathrm{SiN}$ ) cantilevers coated with a thin gold film, which enhances the probe conductivity to ensure a higher field enhancement of the lightning rod effect. However, compared with uncoated probes, gold-coated SiN probes exhibit lower spatial resolution, due to a larger tip radius (greater than $35 \mathrm{~nm}$ ). It was reported that typical spatial resolution of AFM-IR is in the range $50-100 \mathrm{~nm}$ [3]. Furthermore, thin gold-coated SiN cantilevers usually experience substantial conductivity degradation during scanning due to wearing of the gold film at the apex. If the thin gold film is abraded and removed from the tip through surface-tip interaction, it can lose conductivity, resulting in high noise levels or poor signal.

The 'lightning rod effect' has been shown to depend strongly on two other parameters besides the probe material [9]: tip-end radius and incident light polarization. The tip-end radius has been shown to be especially important because the field enhancement is drastically decreased for a rounded tip in comparison with a sharp one. AFM topography imaging is governed by the convolution of the probe tip transfer function with the sample surface. Therefore, the spatial resolution is limited by the finite size of the probe. Up to now, various AFM probe modifications 
have been made to improve imaging resolution using methods such as ion etching [10], plasma etching [11], chemical vapor deposition [12], and focused ion beam milling [13]. Recently, the Belkin group demonstrated the ability to enhance the sensitivity of AFM-IR further by using Au-coated AFM tips on $5 \mathrm{~nm}$ biological thin membranes [14]. However, almost all of these methods are complicated, have a high cost, and have problems with repeatability.

Compared with the above-mentioned methods, the focused electronbeam-induced deposition (EBID) $[15,16]$ process has been developed to be very simple and straight forward. Beard et al [17] used the EBID process to fabricate cylindrical nanoneedle structures on AFM probe tips, which can be used for accurate imaging of surfaces with high, steep features. Brown et al [18] reported the fabrication of electrically conducting, ultra-sharp Pt tip by EBID of platinum organometallic precursor. An advantage of depositing metal nanorod tips through the EBID process is that the probe can remain conductive even after hours of continuous scanning. Up to now, the EBID process shows great potential for precise fabrication of AFM probe tips with specific shape and specific materials, such as platinum [19], tungsten [20], carbon [21], iron [22, 23] cobalt [24] and Au-Fe alloy [25] and with high reliability. Thus, it offers the potential for improved spatial resolution as well as enhanced sensitivity for midinfrared spectral imaging.

In this manuscript, we describe the precise fabrication of sharp metallic AFM-IR probe tip using the focused EBID process, which can produce multiple AFM-IR probe tips one-at-a-time with high repeatability. The EBID process and subsequent electron irradiation were performed in an FEI Helios NanoLab 660. Optimized electron beam voltage, current, deposition time and irradiation time are crucial for determining the radius (12.0 $\pm 5.0 \mathrm{~nm}$ ) and height of the resulting tip, while optimal focus and astigmatism correction are crucial for precise and repeatable production of sharp tips. AFM height images as well as localized nanoIR spectra and chemical IR imaging were recorded in contact mode by using the Anasys nanoIR2 instrument. Two samples were tested to evaluate the durability, spatial resolution and nanoIR signal sensitivity. The first sample is a thin film of vinylidine fluoride co trifluoroethylene, P(VDF-TrFe) with a film thickness around 80-100 nm. The second sample is an epoxy sample embedded with $1 \mu \mathrm{m}$ polystyrene and $3 \mu \mathrm{m}$ polymethyl methacrylate (PMMA) beads, with the film thickness around 200-400 $\mathrm{nm}$. 


\section{Experimental methods}

\subsection{Electron-beam-induced deposition}

We used a dual-beam SEM/FIB microscope (FEI, Helios Nanolab 600) equipped with a Schottky electron emitter for depositing platinum or tungsten probe tips. The Pt precursor, $\mathrm{C}_{5} \mathrm{H}_{4} \mathrm{CH}_{3} \mathrm{Pt}\left(\mathrm{CH}_{3}\right)_{3}$ or $\mathrm{W}$ precursor, $\mathrm{W}(\mathrm{CO})_{6}$ was introduced through a gas-injection needle positioned $\sim 100$ $\mu \mathrm{m}$ from the sample surface. The selection of ideal electron beam voltage, current and duration time, as well as optimal focus and astigmatism correction for EBID process is crucial for determining the tip radius, tip length. Through a series of systematic experiments, Pt probe tips with the smallest radius-of-curvature (RoC) $(12 \pm 5 \mathrm{~nm})$ were grown with the Helios NanoLab 660 microscope via focusing the beam to one spot (spot mode), by setting the electron beam voltage to $18 \mathrm{kV}$ and the beam current of $50 \mathrm{pA}$. To decrease excessive carbon contaminants, the following parameters were used: electron irradiation at $5 \mathrm{keV}$ voltage, $0.4-0.8 \mathrm{nA}$ current, and $1 \mu$ s dwell time.

\subsection{AFM image and localized nanoIR spectrum collection}

A nanoIR2 (Anasys Instruments, Inc.) was used to collect both AFM topography images and localized nanoIR spectra, as well as chemical IR imaging at a constant wavelength. Contact mode nIR2 probes (Model: PR-EX-nIR2, Anasys Instruments) with resonance frequency of $13 \pm 4$ $\mathrm{kHz}$ and spring constant of $0.07-0.4 \mathrm{~N} / \mathrm{m}$ were used [supplementary information, Figure S1(a)], as well as those modified by the EBID process [supplementary information, Figure S1(b)]. The AFM-IR technique is accomplished by coupling a pulsed tunable IR source with an AFM. The pulsed tunable IR source has a pulse length of $\sim 10$ ns and can cover a broad range of the mid-IR region. The light from this source is focused onto the tip-sample contact area. When the pulsed light from the IR source is absorbed by the sample, a rapid heating/expansion of the sample occurs creating an impulse onto the AFM cantilever, which induces an oscillation. 

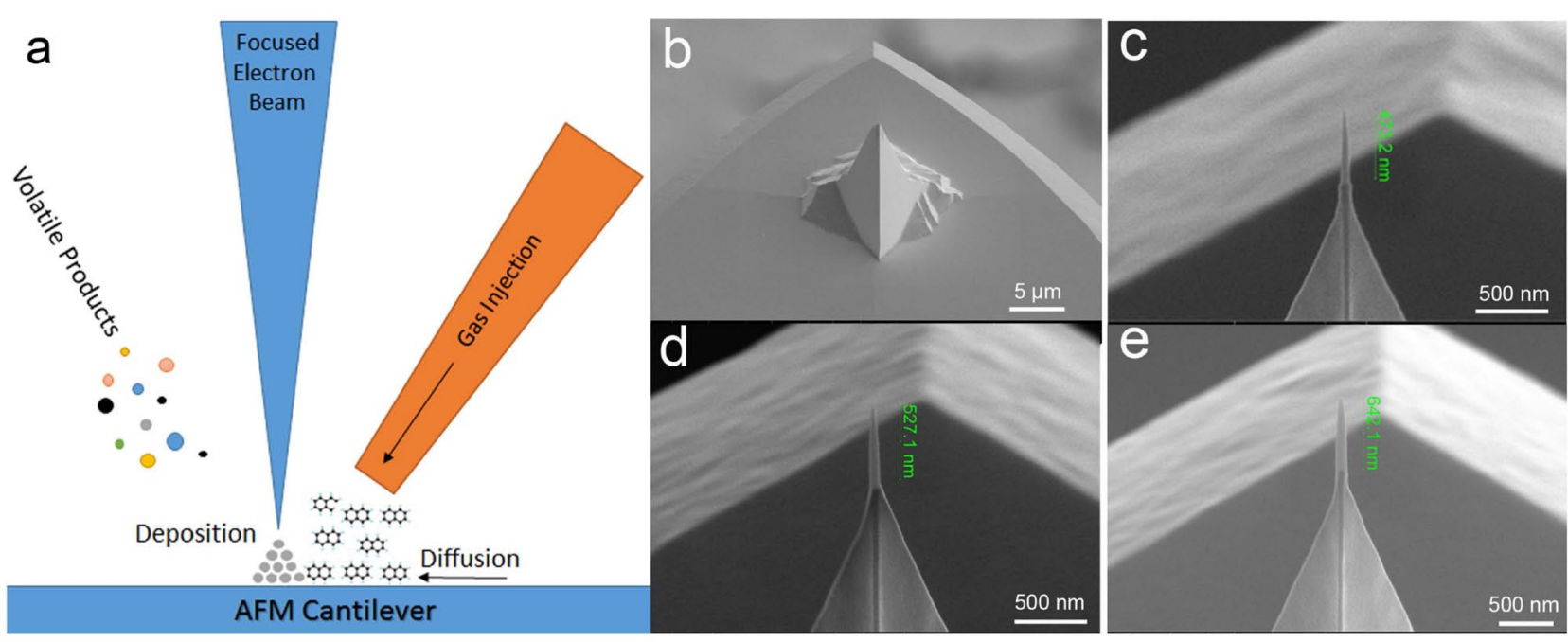

Figure 1. (a) Schematic illustration of the EBID process via spot mode using a Helios NanoLab 660. (b)-(e) shows SEM images of a commercial nIR2 contact probe before (b) and after EBID process of platinum for $5 \mathrm{~s}$ (c), $7 \mathrm{~s} \mathrm{(d),} 10 \mathrm{~s}$ (e) with the tip length of $473.2 \mathrm{~nm}, 527.1 \mathrm{~nm}$ and $642.1 \mathrm{~nm}$, respectively.

\subsection{PVDF thin film preparation}

The PVDF thin film studied here was copolymer of $70 \%$ vinylidine fluoride and $30 \%$ trifluoroethylene, P(VDF-TrFe), made by spin coating. The 70/30 P(VDF-TrFe) was dissolved in methyl ethyl ketene to a concentration of copolymer of $2.5 \mathrm{wt} \%$. First, a $30 \mathrm{~nm}$ thick gold thin film was thermally evaporated onto a $5 \mathrm{~mm}$ by $5 \mathrm{~mm}$ square substrate of ptype silicon with a $300 \mathrm{~nm}$ oxidized layer, in order to enhance the localized IR measurement signal collection. Then a 80-100 nm thick P(VDFTrFe) film was spin coated on the gold surface, followed by drying and annealing at $135^{\circ} \mathrm{C}$ for $4 \mathrm{~h}$ in a vacuum oven.

\section{Results and discussion}

Figure 1(a) shows a schematic diagram of EBID process. Pt deposition is initiated by the interaction of a high energy electron beam with surface-adsorbed organometallic Pt precursor $\mathrm{C}_{5} \mathrm{H}_{4} \mathrm{CH}_{3} \mathrm{Pt}\left(\mathrm{CH}_{3}\right)_{3}$ molecules. An ultra-sharp, high aspect-ratio Pt probe tip was deposited by localizing the beam at a spot on the tip apex of the cantilever, whose tip height was 
controlled by the duration time. This EBID process can easily produce tips with high repeatability and near-100\% yield. Figure 1(b) shows SEM images of a commercial nIR2 contact probe with a stage tilt of $52^{\circ}$. The measured RoC is approximately $\sim 50 \mathrm{~nm}$, as shown in Figure S2(a) (supplementary information) from the top vertical view. Figures 1(c)-(e) shows SEM images of deposited Pt tips after the EBID process for different duration times of $5 \mathrm{~s}$ (c), $7 \mathrm{~s}(\mathrm{~d}), 10 \mathrm{~s}$ (e) respectively. The tip length ranges from $473.2 \mathrm{~nm}$, to $527.1 \mathrm{~nm}$, and to $642.1 \mathrm{~nm}$ accordingly, but all are similar and have a sharp tip radius (RoC $\sim 15 \mathrm{~nm}$ ), as shown in Figure S2(b) (supplementary information) from the top vertical view. The results indicate that increasing duration time produces longer probe tips, but does not increase the tip radius.

However, because the organometallic Pt precursor contains a large amount of carbon, the structure of the deposited Pt tip suffers from carbon contamination. Generally, commercially available Pt precursor $\mathrm{C}_{5} \mathrm{H}_{4} \mathrm{CH}_{3} \mathrm{Pt}\left(\mathrm{CH}_{3}\right)_{3}$ only yields a low Pt content between 10-15 at.\% [26, 27], the resistance of the EBID-modified Pt probe tip was measured between 60 and $220 \mathrm{k} \Omega$ [18]. Such low metal content is the real challenge for near-field enhancement. Recently, it was reported that simple post processing/purification in situ treatment with the electron beam can increase the conductivity 3 orders of magnitude [28-30]. In the present work we performed a subsequent low-energy electron irradiation at $5 \mathrm{keV}, 0.4-0.8 \mathrm{nA}, 1 \mu \mathrm{s}$ dwell time, with 5-10 min duration time, which reduced the carbon composition. Normally, carbon removal takes place during the first few minutes of electron irradiation and contributes to an increase of conductivity, together with graphitization [29]. As a result of carbon removal, the distance between Pt nanocrystal shrinks (volume decreases). Figures 2(a), (b) shows that tip height decreased from 381.1 to $339.2 \mathrm{~nm}$ with electron irradiation of $0.4 \mathrm{nA}$, and $5 \mathrm{~min}$ refresh time. Figures 2(c), (d) shows that the tip height decreased more, from 340.6 to $240.7 \mathrm{~nm}$ with irradiation at $0.8 \mathrm{nA}$ for $10 \mathrm{~min}$ refresh time. It was speculated that the loss of carbon is due to an electron beam-induced reaction of carbon with residual gas molecules (in particular $\mathrm{H}_{2} \mathrm{O}$ and $\mathrm{O}_{2}$ ) present in the SEM chamber at the base pressure of $5 \times 10^{-6} \mathrm{mbar}$ [29]. The energy-dispersive $\mathrm{x}$-ray (EDX) spectrum shows that the Pt/C count ratio increases from 2.1 (before irradiation) to 3.1 (after irradiation at $0.4 \mathrm{nA}$ for $5 \mathrm{~min}$ ) and to 6.7 (after irradiation at $0.8 \mathrm{nA}$ for 10 min). The atomic composition of excessive carbon dropped from 72.27 

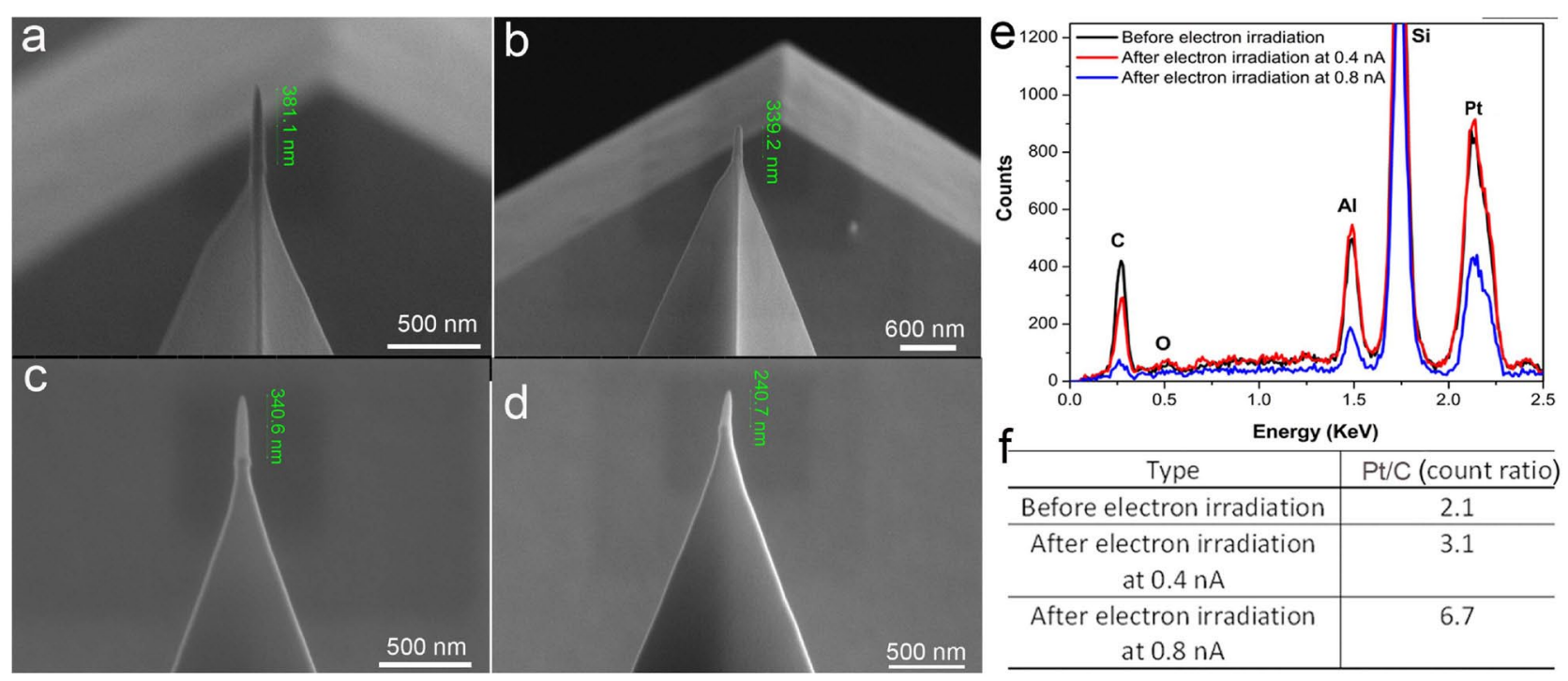

Figure 2. (a)-(d) SEM images of a Pt-deposited probe tip before (a) and after (b) electron irradiation at $0.4 \mathrm{nA}$ for $5 \mathrm{~min}$, as well as before (c) and after (d) electron irradiation at $0.8 \mathrm{nA}$ for $10 \mathrm{~min}$; (e), (f) the corresponding EDX spectra show the Pt/C count ratio before and after electron irradiation.

to 52.02 at.\%, and the atomic composition of platinum increased from 12.21 to 20.25 at.\%. To clarify, all the AFM height images and localized nanoIR spectrum presented in this manuscript were collected by using the tip after electron irradiation.

Poly (vinylidenefluoride) (PVDF) and its copolymers exhibit appealing electrical properties, which have been widely used in organic electronics. The polymer chains have a net dipole moment, pointing from the electronegative fluorine to the electropositive hydrogen, producing a net dipole moment nearly perpendicular to the polymer chain. These chains can crystallize in a quasi-hexagonal close-packed ' $\beta$ - phase' structure with the dipoles of all chains aligned in a structure with maximum polarization. Therefore, the $\beta$-phase is directly related to their ferroelectric, piezoelectric and pyroelectric properties. Recently, there has been an increasing interest in tuning the electrical properties of PVDF by controlling the microstructure down to the nanostructure [31-36]. In this sense, acquiring high-resolution spatial images, high-sensitive localized nanoIR spectra and the corresponding chemical images of $\beta$-phase PVDF at the nanoscale are critical for new applications of ferroelectric PVDF 
polymers. Figures 3(a)-(i) demonstrate the advantages of sharp metallic Pt tips for characterizing these PVDF thin films. The commercial nIR2 probe (Figure 3(a)), and deposited Pt tip (Figure 3(b)) are rounded and sharp, respectively. From Figures 3(c), (e), the AFM height image of PVDF taken by the commercial nIR2 probe (approximately $50 \mathrm{~nm} \mathrm{RoC),} \mathrm{dem-}$ onstrates artifacts because the PVDF grain size is smaller than the probe tip. The resulting image is actually the minimal feature-to-feature variation as the AFM response to topology is dictated by shape of the tip. In contrast, the deposited sharp Pt probe tip (approximately $15 \mathrm{~nm} \mathrm{RoC}$ ) is able to resolve smaller features and the image is more representative of the actual topology of the PVDF membrane surface, as shown in Figures 3(d), (f). Especially while the spatial topography image was zoomed from $3 \mu \mathrm{m} \times 3 \mu \mathrm{m}$ to $1.5 \mu \mathrm{m} \times 1.5 \mu \mathrm{m}$, the resolution of these images can be more clearly compared with Figures 3(e) and (f). Subsequent SEM imaging (Figure S3, supplementary information) confirmed that after a few tens of hours of Pt tip scanning, there was no significant tip wear or contamination. The RoC of the modified Pt tip was almost unchanged as well. The $\mathrm{C}-\mathrm{C}$ antisymmetric stretching, $\mathrm{CF} 2$ antisymmetric stretching, $\mathrm{CH} 2$ waggling and $\mathrm{CH} 2$ bending stretch of all-trans (TT) chains structure at $1071,1176,1398$ and $1428 \mathrm{~cm}^{-1}$, are the fingerprint for $\beta$-phase in the IR spectrum [37]. As shown in Figure 3(g), the nanoIR signal intensity of all these absorption peaks are greatly enhanced for the deposited metallic Pt and sharp tip in comparison with a commercial probe. This Pt modified probe tip enables the potential identification of molecular orientation as well as investigation of the fiber alignment axis. van Bladel [38] studied the near-field enhancement difference between sharp and round edges. His simulation results confirmed that both charge density and field enhancement decrease dramatically at the rounded edge, compared with the case of a sharp edge. In particular, the electromagnetic singularity induced by a tip of radius $20 \mathrm{~nm}$ corresponds to an amplification factor of 13 , but for a tip radius of $80 \mathrm{~nm}$ the amplification factor is only 5. Our experimental results are consistent with these analytical calculations. Furthermore, the topography and corresponding chemical mapping image of PVDF were also recorded and shown in Figures 3(h), (i). We fixed the laser frequency at the absorption peak of 1078 $\mathrm{cm}^{-1}$ and recorded the AFM cantilever deflection amplitude as a function of tip position. Figure 3(i) shows the mapping of the $\mathrm{C}-\mathrm{C}$ antisymmetric stretching absorption band for a scan area of $2 \mu \mathrm{m} \times 1 \mu \mathrm{m}$ of the 


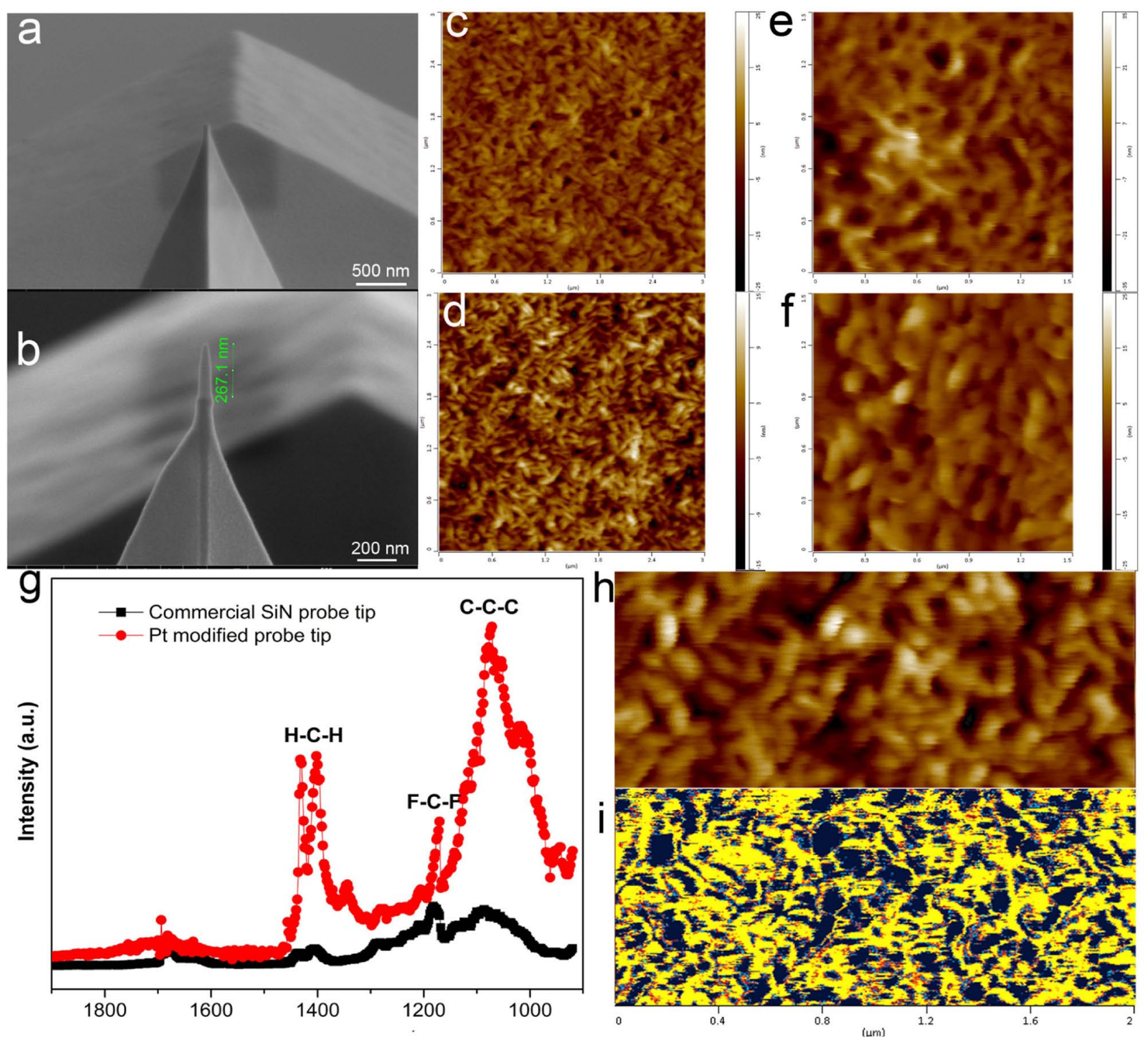

Figure 3. (a), (b) SEM images of a commercial nIR2 contact probe and the same cantilever followed by the EBID process of Pt tip with $267.1 \mathrm{~nm}$ height; (c), (d) AFM height images $(3 \mu \mathrm{m} \times 3 \mu \mathrm{m})$ of PVDF using a commercial nIR2 probe and a modified Pt probe tip respectively; (e), (f) zoom in of height images $(1.5 \mu \mathrm{m} \times 1.5 \mu \mathrm{m})$ by the commercial probe and the modified Pt probe tip respectively; (g) the comparison of localized nanoIR spectra of PVDF between commercial SiN probe and deposited Pt tip; (h), (i) the height image and corresponding chemical mapping image of PVDF at the specific wavelength of $1078 \mathrm{~cm}^{-1}$. 
topographic image (Figure $3(\mathrm{~h})$ ), which allows us to investigate the relationship between morphology and molecular structure of the sample section. As mentioned in the introduction, the Pt-deposited tip through EBID fabrication process is very reliable. Figure S4 (supplementary information) shows a comparison of localized PMMA nanoIR spectrum between commercial SiN tip and two Pt modified tips, which demonstrates that both Pt tips can increase the IR signal intensity.

In addition to the Pt-deposited tips, we also utilized the EBID process to deposit W tips, to compare the effects of different metallic probe materials on the localized nanoIR intensity. Before the $W$ tip was used for AFM-IR measurements, electron irradiation process was performed also to remove the excessive carbon. Figures S5(a), (b) (supplementary information) shows that the deposited $\mathrm{W}$ tip height decreased from 327.9 to $226.6 \mathrm{~nm}$ with electron irradiation of $0.8 \mathrm{nA}$, and $10 \mathrm{~min}$ refresh time. The EDX spectrum shows that the W/C count ratio increases from 3.84 (before irradiation) to 8.93 (after irradiation at $0.8 \mathrm{nA}$ for $10 \mathrm{~min}$ ). The atomic composition of excessive carbon dropped from 79.94 to 39.35 at.\%, and the atomic composition of tungsten increased from 16.83 to 42.26 at.\%. Figures 4(a), (b) shows SEM images of a deposited Pt probe tip with tip length $340.6 \mathrm{~nm}$ and a deposited W probe tip with tip length $460.7 \mathrm{~nm}$ respectively. We noticed that at the same conditions of electron beam voltage and current, the Pt had a larger radius than the $\mathrm{W}$ tip. A possible explanation of this result could be that the platinum contains more light elements, carbon and hydrogen [39], because it is easier to dissociate the $\mathrm{C}_{5} \mathrm{H}_{4} \mathrm{CH}_{3} \mathrm{Pt}\left(\mathrm{CH}_{3}\right)_{3}$ precursors than the $\mathrm{W}(\mathrm{CO})_{6}$ precursor. Thus, the electron mean free paths are longer in the Pt tip than in the $\mathrm{W}$ tip [40]. The AFM height images of epoxy/polystyrene/ PMMA thin film using a deposited Pt probe tip (Figure 4(c)) and deposited W tip (Figure $4(d))$ respectively, show very little difference at the scale of a $10 \mu \mathrm{m} \times$ $10 \mu \mathrm{m}$ scan. However, Figure 4(e) clearly shows the difference of localized nanoIR spectrum of PMMA between the Pt and $\mathrm{W}$ tips. The results indicate that Pt tips are able to generate higher signal intensity compared with W tips. Besides PMMA sample, we also compared results for W-deposited tip with a commercial SiN tip for measuring a PVDF sample, which shows similar results (Figure S6, supplementary information). The $\mathrm{W}$ tip is better than a commercial SiN tip, but not as effective as Pt tip. We speculate that although the deposited electrical resistivity of W $(300 \mu \Omega \cdot \mathrm{cm})$ is lower than $\operatorname{Pt}(600 \mu \Omega \cdot \mathrm{cm})$ in vacuum [40], W is 

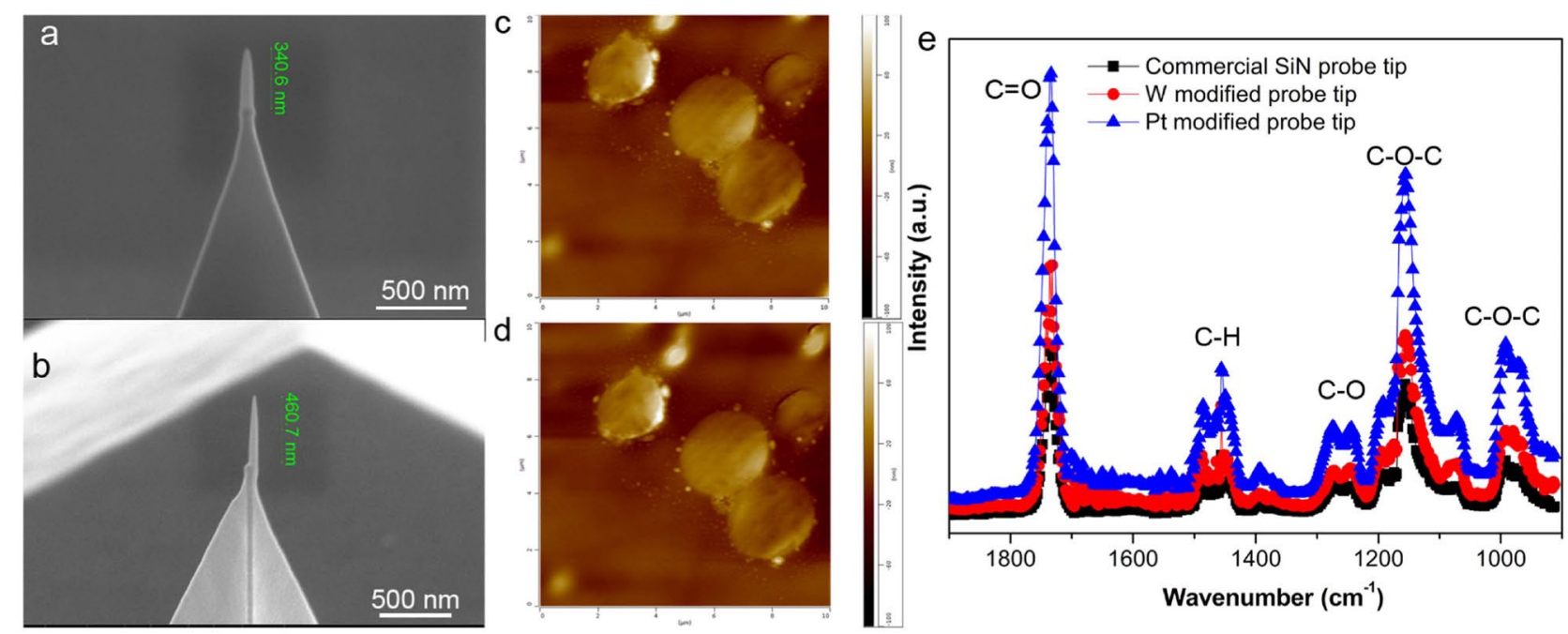

Figure 4. (a), (b) SEM images of deposited Pt and W probe tips, respectively; (c), (d) AFM height images $(10 \mu \mathrm{m} \times 10 \mu \mathrm{m})$ of an epoxy/ polystyrene/PMMA thin film by Pt and $\mathrm{W}$ tip respectively; (e) comparison of localized PMMA nanoIR spectrum between commercial SiN tip, Pt and W tips.

more easily oxidized to $\mathrm{WO}_{3}$ once it is removed from the vacuum chamber, therefore its stability in air is much less than Pt. We believe that both the Pt and W tips by the EBID process have great potential for providing high-resolution height images and high sensitivity chemical images of organic materials. For imaging a thin membrane surface, which requires highly sensitive nanoIR measurement, a relatively conductive Pt tip should be used, so that the molecular structure and orientation can be clearly investigated. Alternatively, if imaging a biological materials, organic materials or polymers with the possible highest resolution is required, an individual short and sharp W tip is ideal. As an example, Figure S7 (supplementary information) shows an AFM height image (2 $\mu \mathrm{m} \times 2 \mu \mathrm{m}$ ) of polystyrene beads with smallest feature size around 20 $\mathrm{nm}$ by using a deposited $\mathrm{W}$ tip.

\section{Conclusion}

In summary, we fabricated sharp, durable, and metallic Pt or $\mathrm{W}$ probe tips with $12.0 \pm 5.0 \mathrm{~nm} \mathrm{RoC}$ by the EBID process. Further electron irradiation can reduce the electrical resistance of deposited probe tips by 
removal of excess carbon contaminants. The EBID approach enables a highly repeatable fabrication of probe tips and is an important step towards making well-defined tips for use in high-resolution and high sensitivity AFM-IR measurements. AFM height imaging and localized nanoIR spectra of PVDF and PMMA thin films were collected to compare the performance of deposited Pt or W probe tips with commercial nIR2 SiN probes. The modified metallic and sharp tips showed better resolution, durability, and sensitivity in generating topography image and localized nanoIR spectrum. Ongoing research will optimize the lightning rod effect in terms of enhancement factor and spatial confinement. We will test more metallic materials to fabricate custom tips, such as gold and silver. We believe that the metallic durable and sharp AFM-IR probe tip via the EBID process will greatly support research associated with polymer science, materials science and life science, especially detailed investigation of structure-chemical property correlations.

Acknowledgments Manufacturing and characterization analysis were performed at the NanoEngineering Research Core Facility (NERCF), which is partially funded by the Nebraska Research Initiative. S S, J S, and S D were supported by the National Science Foundation (NSF) through the Nebraska Materials Research Science and Engineering Center (MRSEC) under Grant No. DMR-1420645, National Key R\&D Program of China (Grant No. 2016YFB0400801), Natural Science Foundation of China (Grant No. 61404147), and the Key Technology Research and Development Program of Jiangsu Province (BE2014147-1).

\section{References}

[1] Dazzi A and Prater C B 2017 AFM-IR: technology and applications in nanoscale infrared spectroscopy and chemical imaging Chem. Rev. 117 5146-73

[2] Katzenmeyer A M, Aksyuk V and Centrone A 2013 Nanoscale infrared spectroscopy: improving the spectral range of the photothermal induced resonance technique Anal. Chem. 85 1972-9

[3] Dazzi A, Prater C B, Hu Q C, Chase D B, Rabolt J F and Marcott C 2012 AFM-IR: combining atomic force microscopy and infrared spectroscopy for nanoscale chemical characterization Appl. Spectrosc. 66 1365-84

[4] Centrone A 2015 Infrared imaging and spectroscopy beyond the diffraction limit Annu. Rev. Anal. Chem. 8 101-26

[5] Dazzi A, Glotin F and Carminati R 2010 Theory of infrared nanospectroscopy by photothermal induced resonance J. Appl. Phys. 107124519 
[6] Novotny L, Bian R X and Xie X S 1997 Theory of nanometric optical tweezers Phys. Rev. Lett. 79 645-8

[7] Goncharenko A V, Wang J K and Chang Y C 2006 Electric near-field enhancement of a sharp semi-infinite conical probe: material and cone angle dependence Phys. Rev. B 74235442

[8] Dazzi A, Saunier J, Kjoller K and Yagoubi N 2015 Resonance enhanced AFM-IR: a new powerful way to characterize blooming on polymers used in medical devices Int. J. Pharm. 484 109-14

[9] Royer P, Barchiesi D, Lerondel G and Bachelot R 2004 Near-field optical patterning and structuring based on local-field enhancement at the extremity of a metal tip Phil. Trans. R. Soc. A 362 821-42

[10] Boisen A, Hansen O and Bouwstra S 1996 AFM probes with directly fabricated tips J. Micromech. Microeng. 6 58-62

[11] Smirnov W, Kriele A, Hoffmann R, Sillero E, Hees J, Williams O A, Yang N J, Kranz $C$ and Nebel C E 2011 Diamond-modified AFM probes: from diamond nanowires to atomic force microscopy-integrated boron-doped diamond electrodes Anal. Chem. 83 4936-41

[12] Cheung C L, Hafner J H, Odom T W, Kim K and Lieber C M 2000 Growth and fabrication with single-walled carbon nanotube probe microscopy tips Appl. Phys. Lett. 76 3136-8

[13] Skibinski J, Rebis J, Wejrzanowski T, Rozniatowski K, Pressard K and Kurzydlowski K J 2014 Imaging resolution of AFM with probes modified with FIB Micron 66 23-30

[14] Lu F, Jin M Z and Belkin M A 2014 Tip-enhanced infrared nanospectroscopy via molecular expansion force detection Nat. Photon. 8 307-12

[15] van Dorp W F and Hagen C W2008 A critical literature review of focused electron beam induced deposition J. Appl. Phys. 104081301

[16] Randolph S J, Fowlkes J D and Rack P D 2006 Focused, nanoscale electronbeam-induced deposition and etching Crit. Rev. Solid State Mater. Sci. 31 55-89

[17] Beard J D and Gordeev S N 2011 Fabrication and buckling dynamics of nanoneedle AFM probes Nanotechnology 22175303

[18] Brown J, Kocher P, Ramanujan C S, Sharp D N, Torimitsu K and Ryan J F 2013 Electrically conducting, ultra-sharp, high aspect-ratio probes for AFM fabricated by electron-beam-induced deposition of platinum Ultramicroscopy 133 62-6

[19] Stanford M G, Lewis B B, Noh J H, Fowlkes J D, Roberts N A, Plank H and Rack P D 2014 Purification of nanoscale electron-beam-induced platinum deposits via a pulsed laser-induced oxidation reaction ACS Appl. Mater. Interfaces 6 21256-63

[20] Henry M R, Kim S and Fedorov A G 2016 High purity tungsten nanostructures via focused electron beam induced deposition with carrier gas assisted supersonic jet delivery of organometallic precursors J. Phys. Chem. C 120 10584-90

[21] Chen I C, Chen L H, Orme C, Quist A, Lal R and Jin S H 2006 Fabrication of highaspect-ratio carbon nanocone probes by electron beam induced deposition patterning Nanotechnology 17 4322-6 
[22] Gavagnin M, Wanzenboeck H D, Belic D, Shawrav M M, Persson A, Gunnarsson K, Svedlindh P and Bertagnolli E 2014 Magnetic force microscopy study of shape engineered FEBID iron nanostructures Phys. Status Solidi a 211 368-74

[23] Gavagnin M, Wanzenboeck H D, Shawrav M M, Belic D, Wachter S, Waid S, Stoeger-Pollach M and Bertagnolli E 2014 Focused electron beam-induced CVD of iron: a practical guide for direct writing Chem. Vapor Depos. 20 243-50

[24] Sangiao S, Magen C, Mofakhami D, de Loubens G and De Teresa J M 2017 Magnetic properties of optimized cobalt nanospheres grown by focused electron beam induced deposition (FEBID) on cantilever tips Beilstein J. Nanotechnol. 8 2106-15

[25] Shawrav M M, Belic D, Gavagnin M, Wachter S, Schinnerl M, Wanzenboeck H D and Bertagnolli E 2014 Electron beam-induced CVD of nanoalloys for nanoelectronics Chem. Vapor Depos. 20 251-7

[26] Botman A, Mulders J J L and Hagen C W 2009 Creating pure nanostructures from electron-beam-induced deposition using purification techniques: a technology perspective Nanotechnology 20372001

[27] Rotkina L, Lin J F and Bird J P 2003 Nonlinear current- voltage characteristics of Pt nanowires and nanowire transistors fabricated by electron-beam deposition Appl. Phys. Lett. 83 4426-8

[28] Plank H, Kothleitner G, Hofer F, Michelitsch S G, Gspan C, Hohenau A and Krenn J R 2011 Optimization of postgrowth electron-beam curing for focused electronbeam-induced Pt deposits J. Vac. Sci. Technol. B 29051801

[29] Porrati F, Sachser R, Schwalb C H, Frangakis A S and Huth M 2011 Tuning the electrical conductivity of Pt-containing granular metals by postgrowth electron irradiation J. Appl. Phys. 109063715

[30] Plank H, Gspan C, Dienstleder M, Kothleitner G and Hofer F 2008 The influence of beam defocus on volume growth rates for electron beam induced platinum deposition Nanotechnology 19485302

[31] Cauda V, Canavese G and Stassi S 2015 Nanostructured piezoelectric polymers J. Appl. Polym. Sci. 13241667

[32] Hu Z J, Tian M W, Nysten B and Jonas A M 2009 Regular arrays of highly ordered ferroelectric polymer nanostructures for non-volatile low-voltage memories Nat. Mater. 8 62-7

[33] Song J F, Lu H D, Foreman K, Li S M, Tan L, Adenwalla S, Gruverman A and Ducharme S 2016 Ferroelectric polymer nanopillar arrays on flexible substrates by reverse nanoimprint lithography J. Mater. Chem. C 4 5914-21

[34] Song J F, Lu H D, Li S M, Tan L, Gruverman A and Ducharme S 2016 Fabrication of ferroelectric polymer nanostructures on flexible substrates by soft-mold reverse nanoimprint lithography Nanotechnology 27015302

[35] Wang X D et al 2016 Flexible graphene field effect transistor with ferroelectric polymer gate Opt. Quantum Electron. 48345

[36] Dan L et al 2018 The ambipolar evolution of a high-performance $\mathrm{WSe}_{2}$ transistor assisted by a ferroelectric polymer Nanotechnology 29105202 
[37] Kobayashi M, Tashiro K and Tadokoro H 1975 Molecular vibrations of 3 crystal forms of Poly(vinylidene fluoride) Macromolecules 8 158-71

[38] van Bladel J 1995 Singular Electromagnetic Fields and Sources (Oxford: IEEE Press)

[39] Marzi G D, Iacopino D, Quinn A J and Redmond G 2004 Probing intrinsic transport properties of single metal nanowires: direct-write contact formation using a focused ion beam J. Appl. Phys. 96 3458-62

[40] Reguer A, Bedu F, Tonneau D, Dallaporta H, Prestigiacomo M, Houel A and Sudraud P 2008 Structural and electrical studies of conductive nanowires prepared by focused ion beam induced deposition J. Vac. Sci. Technol. B 26 175-80

[19] Stanford M G, Lewis B B, Noh J H, Fowlkes J D, Roberts N A, Plank H and Rack P D 2014 Purification of nanoscale electron-beam-induced platinum deposits via a pulsed laser-induced oxidation reaction ACS Appl. Mater. Interfaces 6 21256-63

[20] Henry M R, Kim S and Fedorov A G 2016 High purity tungsten nanostructures via focused electron beam induced deposition with carrier gas assisted supersonic jet delivery of organometallic precursors J. Phys. Chem. C 120 10584-90

[21] Chen I C, Chen L H, Orme C, Quist A, Lal R and Jin S H 2006 Fabrication of highaspect-ratio carbon nanocone probes by electron beam induced deposition patterning Nanotechnology 17 4322-6

[22] Gavagnin M, Wanzenboeck H D, Belic D, Shawrav M M, Persson A, Gunnarsson K, Svedlindh P and Bertagnolli E 2014 Magnetic force microscopy study of shape engineered FEBID iron nanostructures Phys. Status Solidi a 211 368-74

[23] Gavagnin M, Wanzenboeck H D, Shawrav M M, Belic D, Wachter S, Waid S, Stoeger-Pollach M and Bertagnolli E 2014 Focused electron beam-induced CVD of iron: a practical guide for direct writing Chem. Vapor Depos. 20 243-50

[24] Sangiao S, Magen C, Mofakhami D, de Loubens G and De Teresa J M 2017 Magnetic properties of optimized cobalt nanospheres grown by focused electron beam induced deposition (FEBID) on cantilever tips Beilstein J. Nanotechnol. 8 2106-15

[25] Shawrav M M, Belic D, Gavagnin M, Wachter S, Schinnerl M, Wanzenboeck H D and Bertagnolli E 2014 Electron beam-induced CVD of nanoalloys for nanoelectronics Chem. Vapor Depos. 20 251-7

[26] Botman A, Mulders J J L and Hagen C W 2009 Creating pure nanostructures from electron-beam-induced deposition using purification techniques: a technology perspective Nanotechnology 20372001

[27] Rotkina L, Lin J F and Bird J P 2003 Nonlinear current- voltage characteristics of Pt nanowires and nanowire transistors fabricated by electron-beam deposition Appl. Phys. Lett. 83 4426-8

[28] Plank H, Kothleitner G, Hofer F, Michelitsch S G, Gspan C, Hohenau A and Krenn J R 2011 Optimization of postgrowth electron-beam curing for focused electronbeam-induced Pt deposits J. Vac. Sci. Technol. B 29051801 
[29] Porrati F, Sachser R, Schwalb C H, Frangakis A S and Huth M 2011 Tuning the electrical conductivity of Pt-containing granular metals by postgrowth electron irradiation J. Appl. Phys. 109063715

[30] Plank H, Gspan C, Dienstleder M, Kothleitner G and Hofer F 2008 The influence of beam defocus on volume growth rates for electron beam induced platinum deposition Nanotechnology 19485302

[31] Cauda V, Canavese G and Stassi S 2015 Nanostructured piezoelectric polymers J. Appl. Polym. Sci. 13241667

[32] Hu Z J, Tian M W, Nysten B and Jonas A M 2009 Regular arrays of highly ordered ferroelectric polymer nanostructures for non-volatile low-voltage memories Nat. Mater. 8 62-7

[33] Song J F, Lu H D, Foreman K, Li S M, Tan L, Adenwalla S, Gruverman A and Ducharme S 2016 Ferroelectric polymer nanopillar arrays on flexible substrates by reverse nanoimprint lithography J. Mater. Chem. C 4 5914-21

[34] Song J F, Lu H D, Li S M, Tan L, Gruverman A and Ducharme S 2016 Fabrication of ferroelectric polymer nanostructures on flexible substrates by soft-mold reverse nanoimprint lithography Nanotechnology 27015302

[35] Wang X D et al 2016 Flexible graphene field effect transistor with ferroelectric polymer gate Opt. Quantum Electron. 48345

[36] Dan L et al 2018 The ambipolar evolution of a high-performance $\mathrm{WSe}_{2}$ transistor assisted by a ferroelectric polymer Nanotechnology 29105202

[37] Kobayashi M, Tashiro K and Tadokoro H 1975 Molecular vibrations of 3 crystal forms of Poly(vinylidene fluoride) Macromolecules 8 158-71

[38] van Bladel J 1995 Singular Electromagnetic Fields and Sources (Oxford: IEEE Press)

[39] Marzi G D, Iacopino D, Quinn A J and Redmond G 2004 Probing intrinsic transport properties of single metal nanowires: direct-write contact formation using a focused ion beam J. Appl. Phys. 96 3458-62

[40] Reguer A, Bedu F, Tonneau D, Dallaporta H, Prestigiacomo M, Houel A and Sudraud P 2008 Structural and electrical studies of conductive nanowires prepared by focused ion beam induced deposition J. Vac. Sci. Technol. B 26 175-80 


\section{Supplementary Information}

\section{Focused Electron-Beam-Induced Deposition for Fabrication of Highly Durable and Sensitive Metallic AFM-IR Probes}

Wen Qian ${ }^{1}$, Shuo Sun ${ }^{2,3}$, Jingfeng Song ${ }^{2}$, Charles Nguyen ${ }^{1}$, Stephen Ducharme ${ }^{2}$ and Joseph A. Turner ${ }^{1}$

${ }^{1}$ Department of Mechanical \& Materials Engineering, University of Nebraska-Lincoln, Lincoln, NE 68588

${ }^{2}$ Department of Physics \& Astronomy and Nebraska Center for Materials and Nanoscience, University of Nebraska-Lincoln, Lincoln, NE 68588

3 National Laboratory for Infrared Physics, Shanghai Institute of Technical Physics, Chinese Academy of Sciences, Yu Tian Road 500, Shanghai 200083, China

Correspondence Email: wqian2@unl.edu and jaturner@unl.edu
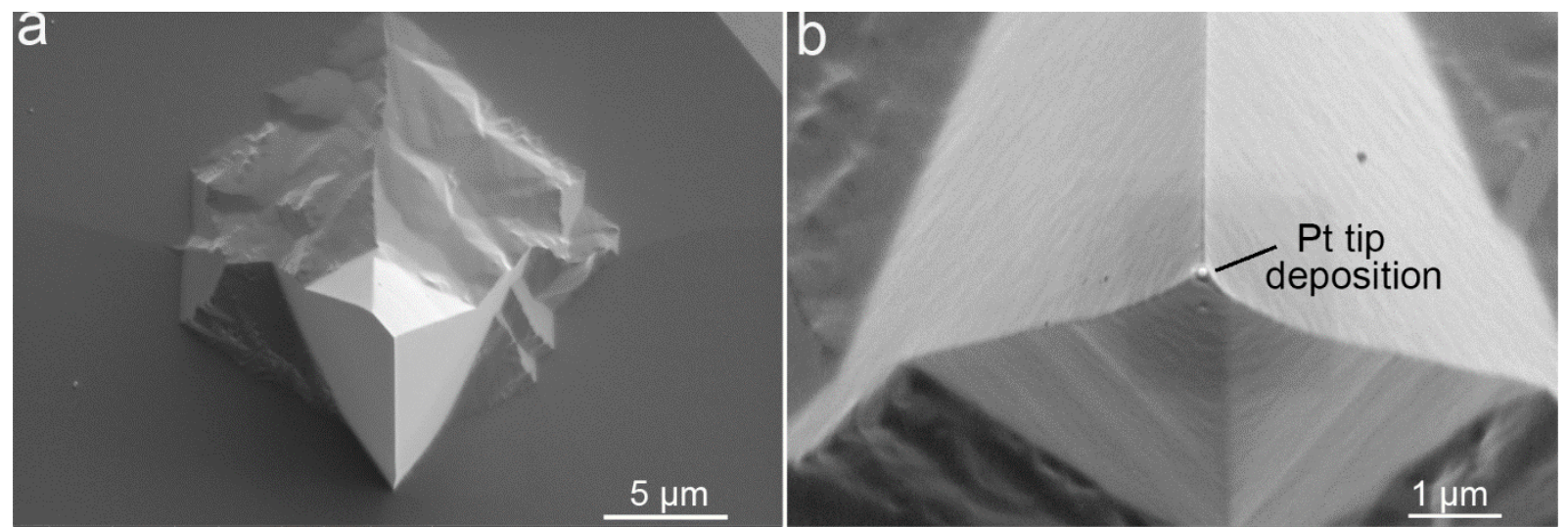

Figure S1. SEM images of a commercial Anasys nIR2 SiN probe (a) and after the subsequent EBID process to deposit a Pt tip (b) showing the perpenticular point of view.
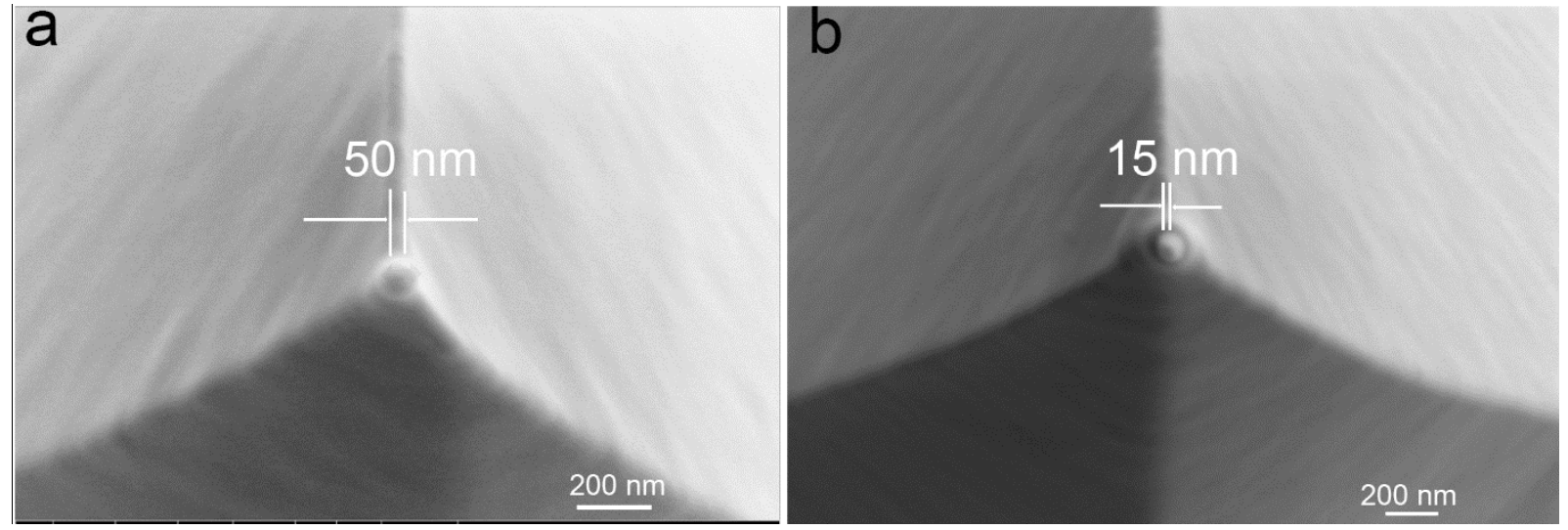

Figure S2. High magnification SEM images of commercial Anasys nIR2 SiN probe (a) and after subsequent EBID process deposition of Pt tip (b) showing the perpendicular point of view. 

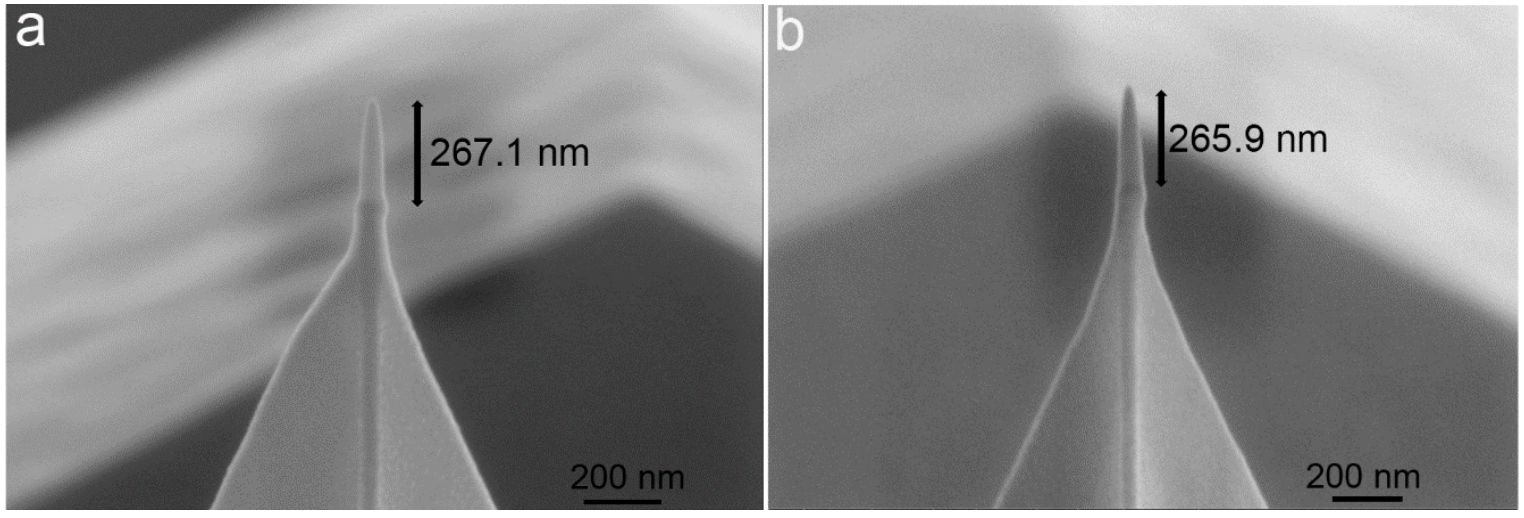

Figure S3. Pt deposited tip before scanning (a, $267.1 \mathrm{~nm}$ tip length) and after scanning for a few tens of hours (b, $265.9 \mathrm{~nm}$ tip length).

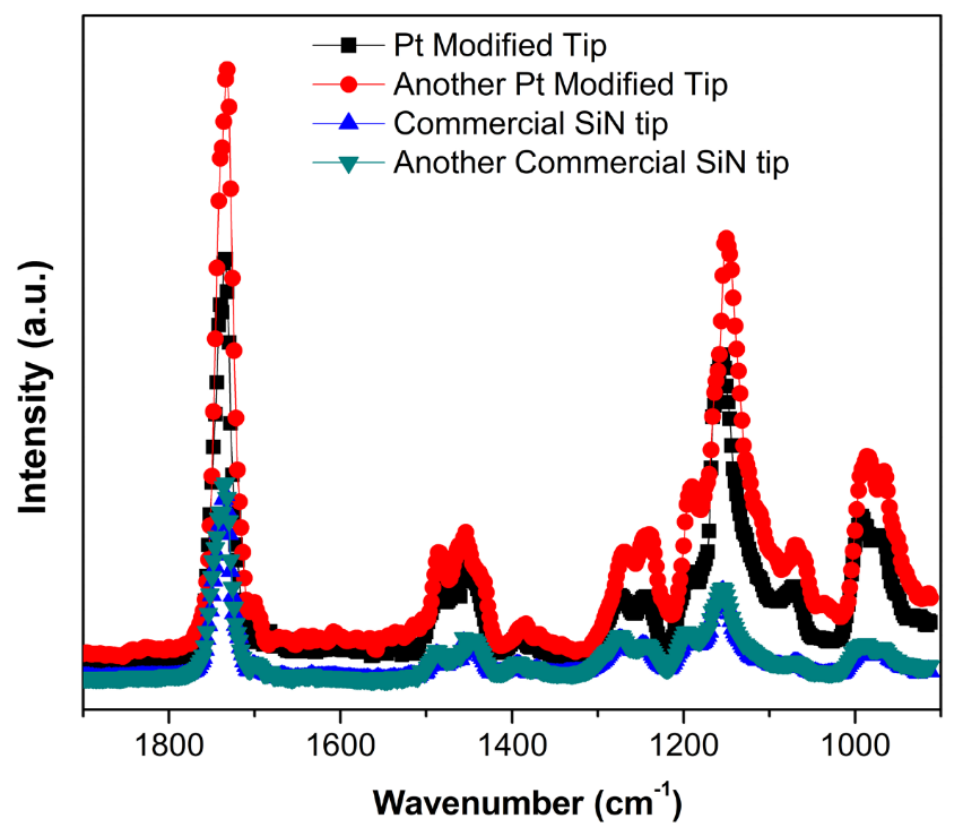

Figure S4. Comparison of localized PMMA nanoIR spectrum between commercial SiN tip and two Pt modified tip. 

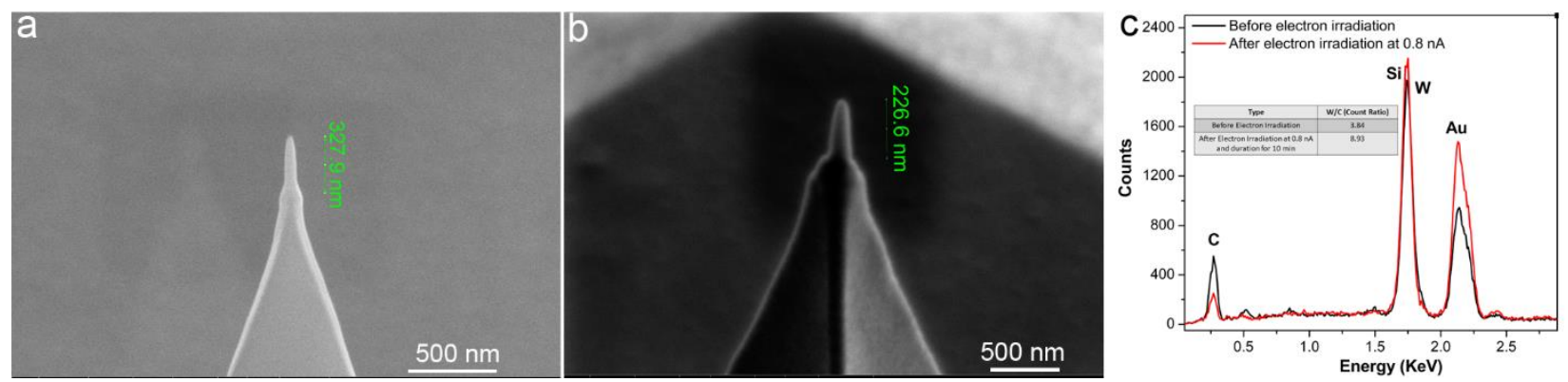

Figure S5. (a-b) SEM images of W-deposited probe tip before (a) and after (b) electron irradiation at 0.8 $\mathrm{nA}$ for 10 mins; (c) the corresponding EDX spectra shows W/C count ratio before and after electron irradiation.

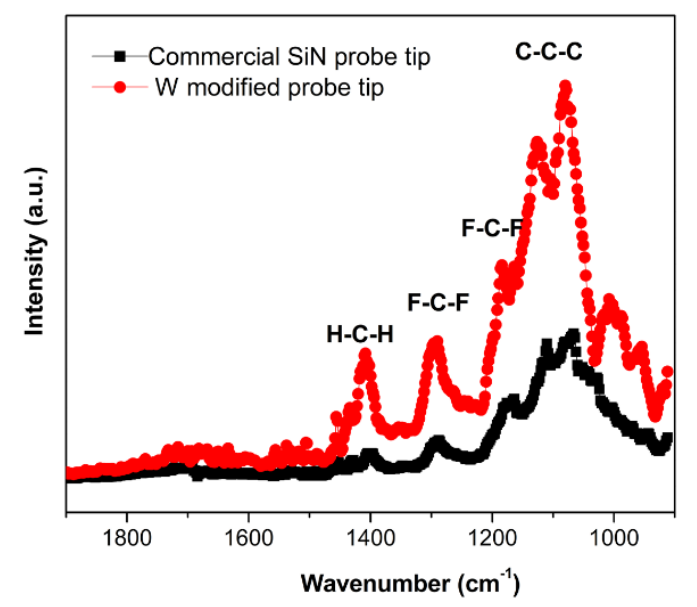

Figure S6. Comparison of localized nanoIR spectra of PVDF between commercial SiN probe and W modified probe tip.

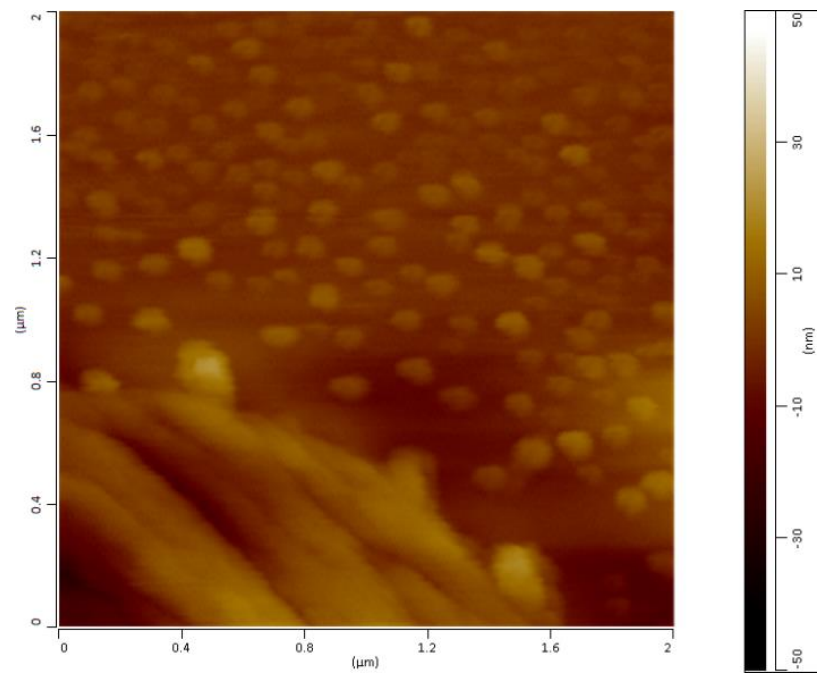

Figure S7. AFM height image $(2 \mu \mathrm{m} * 2 \mu \mathrm{m})$ of polystyrene beads with smallest feature size around 20 $\mathrm{nm}$ using a modified $\mathrm{W}$ probe tip. 